\title{
Long-term progression of visual field defects and related factors in medically treated normal tension glaucoma
}

This article was published in the following Dove Press journal:

Clinical Ophthalmology

\author{
Keiji Yoshikawa' \\ Kazunori Santo ${ }^{2}$ \\ Hiroko Hizaki² \\ Masayo Hashimoto ${ }^{2}$ \\ 'Yoshikawa Eye Clinic, Tokyo, ${ }^{2}$ Medical \\ Affairs, Santen Pharmaceutical Co. \\ Ltd., Osaka, Japan
}

Purpose: To analyze factors related to long-term progression of visual field defects (VFD) in patients with normal tension glaucoma (NTG) under medical therapy.

Patients and methods: Clinical data from 622 eyes of 311 primary open-angle glaucoma and NTG patients were collected from April 2006 to March 2016. Of these patients, those with normal intraocular pressure (IOP); glaucomatous VFD judged by Anderson's criteria; corrected visual acuity $\geq 0.7$; receiving more than 6 years medical therapy; having undergone $\geq 10$ visual field tests performed at 6-month intervals using a Humphrey field analyzer (Swedish Interactive Threshold Algorithms standard, C 24-2 program); and having reliability coefficients of visual field testing $<33 \%$ and mean deviation (MD) more than -20 decibels in the initial visual field test were included in data analysis. The relationship between MD slope deterioration at final observation and consecutive decreases in MD value during the observation period, as well as clinical characteristics and IOP-related factors, were analyzed.

Results: Of 134 eyes in 134 NTG patients meeting all eligibility criteria, significant MD slope deterioration was observed in 80 eyes (59.7\%). MD slope deterioration was significantly associated with consecutive decreases in MD values (Cochran-Armitage trend test: $P=0.0000$; univariate logistic regression analysis: $P<0.0001)$. While no significant relationship was observed between central corneal thickness, refractive error, or prevalence of disc hemorrhage, consecutive decreases in MD value was significantly related to MD slope deterioration (univariate logistic regression analysis: $P<0.0001$ ). A reduction of IOP during the follow-up period was significantly related to nondeterioration of the MD slope (multivariate logistic regression analysis: $P=0.0020$ )

Conclusion: In this 6-year observation of NTG patients treated with medical therapy, the occurrence of three or more consecutive decreases in MD value was significantly associated with visual field deterioration. Reduction in IOP was postulated to be contributing in the prevention of VFD progression.

Keywords: long-term follow-up, MD slope deterioration, consecutive decrease in MD value, intraocular pressure reduction

\section{Introduction}

Standard automated perimetry is typically employed for the assessment of glaucomatous visual field defects (VFD), as it provides a quantitative, objective assessment of the severity and progression of VFD. ${ }^{1}$ In order to manage glaucoma effectively, it is critical to detect the progression of VFD in its earliest stages; however, this strategy requires frequent repeat testing of visual fields. ${ }^{2,3}$ We have previously reported that three or more consecutive decreases in mean deviation (MD) could be an indicator of 
the early progression of VFD detected at the final observation in patients with primary open-angle glaucoma (POAG) and normal tension glaucoma (NTG). ${ }^{4}$

Reductions in intraocular pressure (IOP) may prevent visual field deterioration. ${ }^{4}$ While decrease in IOP has been effective in preventing VFD progression in POAG eyes, ${ }^{5-7}$ a few studies have reported its contribution to preventing long-term VFD progression in NTG eyes. ${ }^{8-11}$ Accordingly, we focused on NTG patients who had been treated with medical therapy and followed for at least 6 years and analyzed the relationship between MD slope at final observation and consecutive decreases in MD values over time. We also assessed several factors that may affect VFD progression, such as disc hemorrhage (DH) ${ }^{12}$ refractive error, ${ }^{9}$ and central corneal thickness (CCT), ${ }^{13}$ which were not investigated in our previous report.

\section{Patients and methods}

\section{Study design}

This was a retrospective study.

\section{Patients}

This study was conducted in compliance with the Declaration of Helsinki and Ethical Guidelines for Medical and Health Research Involving Human Subjects in Japan. All participants provided written informed consent for data collection at the time of their initial visit. Prior to the present study, we gave the opportunity to participants to opt out of the study.

From April 2006 through March 2016, data were collected from 311 POAG and NTG patients undergoing treatment with topical glaucoma medications. Patients who had undergone routine ophthalmologic examinations during the follow-up period, including measurement of IOP and visual fields, were included in the study. Eligible patients fulfilled all of the following criteria: 1) diagnosis of NTG at baseline; 2) age 20 years or older at the start of the follow-up period; 3) measurement of baseline IOP by Goldmann applanation tonometry before the start of treatment; 4) testing of visual fields with a Humphrey field analyzer (Zeiss-Humphrey, San Leandro, CA, USA) without alteration of the strategy (Swedish Interactive Threshold Algorithms standard) or $\mathrm{C}$ 24-2 program; 5) accurately measurable IOP (excluding those with history of keratorefractive surgery, ocular injury, and keratoconus); 6) no history or complication of apparent retinal disease; and 7) MD greater than -20 decibels ( $\mathrm{dB}$ ) during visual field testing at the start of the follow-up period.

As parameters of VFD progression, MD slope deterioration and consecutive decreases in MD value were calculated using data from reliable visual field tests for more than 6 years
( $\geq 10$ times at 6-month intervals) meeting the following two criteria: 1) fixation losses $<33 \%$; and 2) false-positive or false-negative $<33 \%$. Patients with a history of glaucoma surgery with a corrected visual acuity of $<0.7$ were excluded. This study involved a single eye per patient. If both eyes satisfied all criteria, the eye with a lower MD value was included. There were no restrictions on the type or number of topical glaucoma medications used, and patients of both sexes were enrolled. Ultimately, 134 eyes of 134 patients met all eligibility criteria.

The study protocol was approved by the Institutional Review Board of Kyoyukai RiverSide Clinic in Sapporo, Japan. This study was registered to UMIN (ID: UMIN000022334) in advance of data collection.

\section{Investigations}

MD values and MD slope were calculated with the use of HfaFiles Version 5.0 software (Beeline, Tokyo, Japan). As previously reported, "deterioration of MD slope" was defined if the MD slope showed significantly $(P<0.05)$ negative value calculated by the least-square method at the final visual field test. " ${ }^{4}$ "Deterioration of MD value" was defined as a decrease in the MD value by more than $0.05 \mathrm{~dB}$ between two consecutive tests. "Consecutive deterioration" was defined as a successive decrease in MD values between follow-up visits. Similarly, "triple deterioration" and "quadruple deterioration" were defined as three or four consecutive decreases in MD value, respectively. The largest number of consecutive decreases was used for analysis.

In advance of initiation of treatment, IOP was measured four or more times, and baseline IOP was defined as the mean value of all IOP values excluding the first IOP measurement value. For the purposes of this study, "mean IOP" was defined as the mean of all IOP values measured every 3 months during the follow-up period. IOP fluctuation was calculated as the standard deviation (SD) of the mean follow-up IOP, and the maximum and minimum IOP during the follow-up period were also determined. The difference between baseline IOP and mean IOP was defined as $\triangle \mathrm{IOP}$, and the IOP reduction rate was determined by calculating $\triangle I O P$ as a percentage of baseline IOP. DH was assessed by ophthalmoscopy (Kowa, Nagoya, Japan) at each visit, and fundus photography (Kowa) was performed every 6 months. CCT was measured with Cirrus HD-OCT 400 Version 5.1 (Zeiss-Humphrey).

\section{Statistical analysis}

Patients' identifying information was concealed before data entry. The Cochran-Armitage trend test and logistic regression analysis were performed to analyze the relationship 
between MD slope and consecutive decreases in MD value.

Clinical characteristics (such as prevalence of $\mathrm{DH}$ ) and IOP-related factors were compared between the two groups patients with deterioration of MD slope and those without deterioration - using the Welch's $t$-test, Fisher's exact test, or the Wilcoxon rank-sum test depending on the scale of the data. The relationship between various factors and the deterioration of MD slope were evaluated with multivariate logistic regression analysis using the presence or absence of MD slope deterioration as the objective variable and the consecutive decrease of MD values or the IOP reduction rate versus baseline as explanatory variables.

Analyses were performed with EZR Version 1.32 software (Saitama Medical Center, Jichi Medical University, Saitama, Japan), which provides a graphical user interface for R (The R Foundation for Statistical Computing, Vienna, Austria). The level of significance was set at 5\% (two-sided). All analyses were inspected by Satista Co. Ltd. (https://www. satista.jp/medical//).

\section{Results}

Of 622 eyes of 311 POAG and NTG patients, 134 eyes from 134 NTG patients fulfilled all eligibility criteria, consisting of 82 female and 52 male patients with a mean \pm SD age of $57.7 \pm 11.3$ years (range, $28-80$ years; all results are given as mean $\pm \mathrm{SD}$ [range] unless otherwise indicated). The follow-up period was $8.05 \pm 0.82$ years (6-12 years). Baseline IOP was $14.96 \pm 1.98 \mathrm{mmHg}(10.7-20.0 \mathrm{mmHg})$; refractive error was $-3.19 \pm 3.56$ diopters (D) $(-13.3$ to $+6.8 \mathrm{D})$; and CCT was $520.5 \pm 32.7 \mu \mathrm{m}(440-606 \mu \mathrm{m})$. The initial MD value was $-7.46 \pm 5.53 \mathrm{~dB}(-19.11$ to $+1.15 \mathrm{~dB})$, and the final MD value was $-10.35 \pm 6.49 \mathrm{~dB}(-26.39$ to $+0.26 \mathrm{~dB})($ Table 1$)$.

During the observation period, a consecutive decrease in MD values was observed in $97 \%$ of the eyes (130/134). Among these, a triple or quadruple consecutive decrease in MD values was observed in $54.5 \%$ of eyes (73/134) (Figure 1). We found significant deterioration of MD slope at the final observation in $59.7 \%$ of eyes $(80 / 134)$; the mean change in MD slope was $-0.22 \pm 0.22 \mathrm{~dB} / \mathrm{yr}$ (Figure 2 ). A consecutive decrease in MD values was significantly correlated with deterioration of $\mathrm{MD}$ slope, both by trend analysis $(P=0.0000)$ and univariate logistic regression analysis (odds ratio: $2.19 ; 95 \%$ confidence interval [CI]: 1.44-3.34; $P<0.0001$ ) (Table 2).

Mean IOP during the observation period was $12.01 \pm 0.88$ $\mathrm{mmHg}(8.8-15.0 \mathrm{mmHg})$, and mean IOP fluctuation was $0.97 \pm 0.22 \mathrm{mmHg}(0.6-2.0 \mathrm{mmHg})$. The IOP reduction rate relative to baseline was $18.82 \% \pm 8.48 \%(-0.3 \%$ to $35.9 \%)$. DH was observed in $21.6 \%$ of eyes $(29 / 134)$.
Table I Patient characteristics, IOP data, and visual field data

\begin{tabular}{lll}
\hline Characteristics & $\mathbf{n}(\%)$ & Mean \pm SD \\
\hline Sex & & \\
$\quad$ Female & $82(61.2)$ & \\
$\quad$ Male & $52(38.8)$ & $57.7 \pm 11.3$ \\
Age (years) & & $8.05 \pm 0.82$ \\
Follow-up period (years) & $-3.19 \pm 3.56$ \\
Refractive error (D) & $520.5 \pm 32.7$ \\
CCT ( $\mu$ m) & \\
DH & & \\
$\quad+$ & $29(21.6)$ & \\
$\quad-$ & $105(78.4)$ & $14.96 \pm 1.98$ \\
Baseline IOP $(\mathrm{mmHg})$ & & $12.01 \pm 0.88$ \\
Mean IOP (mmHg) & $18.82 \pm 8.48$ \\
IOP reduction rate $(\%)$ & $-2.95 \pm 1.60$ \\
IOP reduction range $(\mathrm{mmHg})$ & & $14.32 \pm 1.63$ \\
Maximum IOP $(\mathrm{mmHg})$ & $10.29 \pm 0.98$ \\
Minimum IOP $(\mathrm{mmHg})$ & $4.03 \pm 1.27$ \\
IOP range (mmHg) & & $0.97 \pm 0.22$ \\
IOP fluctuation $(\mathrm{mmHg})$ & $-7.46 \pm 5.53$ \\
Initial MD value $(\mathrm{dB})$ & & $-10.35 \pm 6.49$ \\
Final MD value $(\mathrm{dB})$ & & $-0.22 \pm 0.22$ \\
MD slope (dB/yr) & & \\
\hline
\end{tabular}

Notes: Mean \pm SD number of visual field tests: $15.7 \pm 3.6$ (range, II-29). Mean \pm SD number of IOP measurements: $37.8 \pm 4.4$ (range, 23-4I).

Abbreviations: CCT, central corneal thickness; DH, disc hemorrhage; IOP, intraocular pressure; MD, mean deviation; SD, standard deviation.

No significant differences in clinical characteristics were evident between groups with or without MD slope deterioration. Although there was no significant difference in initial and observation period MD values between the two groups, MD slope was significantly steeper in the MD slope deterioration group than in the nondeterioration group $(-0.32 \mathrm{~dB} /$ yr versus $-0.06 \mathrm{~dB} / \mathrm{yr} ; P<0.0001)$.

There were no significant differences in the prevalence of DH, degree of refractive error, and CCT between the two groups. However, the IOP reduction rate was significantly greater in the nondeterioration group than in the MD slope deterioration group $(P=0.0053)$ (Table 3$)$. Multivariate analysis revealed that the number of consecutive decreases in MD value was significantly associated with deterioration of the MD slope (odds ratio: $2.43 ; 95 \%$ CI: $1.55-3.80 ; P<0.0001$ ), while IOP reduction rate was significantly associated with nondeterioration of MD slope (odds ratio: 0.92; 95\% CI: 0.88-0.97; $P=0.0020$ ) (Table 4).

\section{Discussion}

During the 6-year follow-up period of the present study, the occurrence of three or more consecutive decreases in MD values was significantly related to MD slope deterioration in patients with NTG who were taking topical glaucoma medications. In contrast, reduction of IOP may play a role in the nondeterioration of MD slope. 


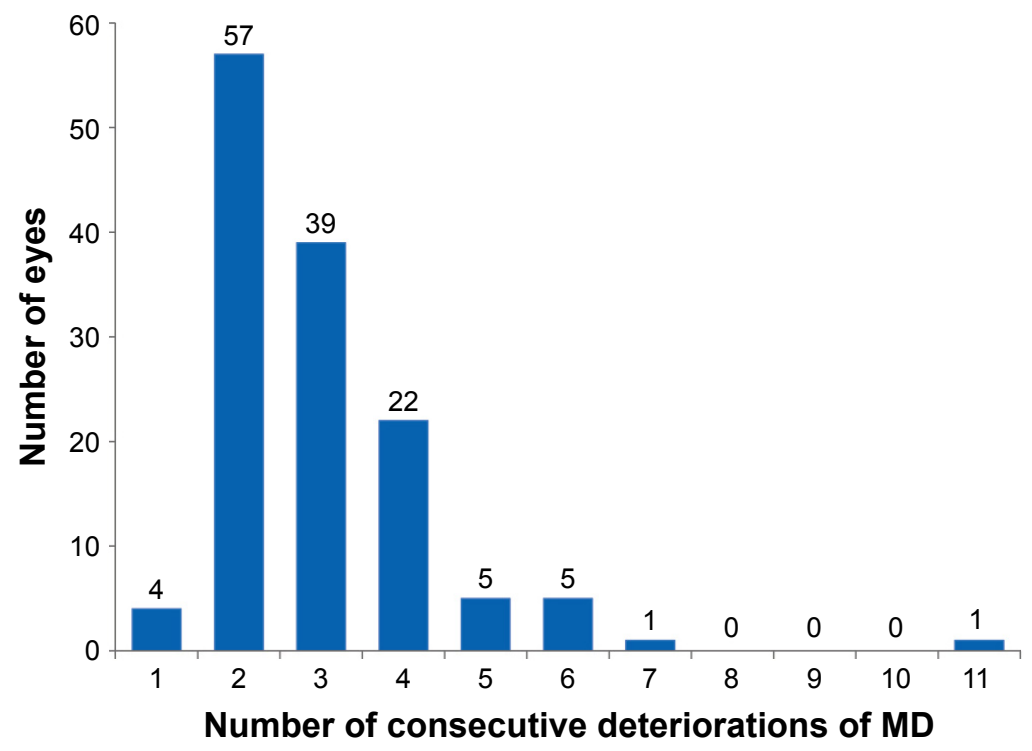

Figure I Distribution of number of eyes in which consecutive decrease in MD value was observed.

Notes: Of 130 eyes in which consecutive decrease in MD value was observed during the follow-up period, the number of two consecutive was 57 eyes (43.8\%). Three consecutive and four or more consecutive was observed in 39 eyes $(30.0 \%)$ and 34 eyes $(26.2 \%)$, respectively.

Abbreviation: MD, mean deviation.

In a previous study, we reported that three or more consecutive decreases in MD during follow-up is a risk factor for significant deterioration of MD slope in patients with POAG and NTG, and that eyes with MD slope nondeterioration had significantly higher rates of reduction in IOP. ${ }^{4}$ However, our previous study included POAG patients in whom a decrease of abnormally elevated IOP is likely to prevent VFD progression; in contrast, IOP levels are within the normal range in NTG patients. In the present study, we examined a variety of factors that may affect VFD progression in patients with NTG.

Significant deterioration of the MD slope occurred in $59.3 \%$ of patients with NTG. Previous long-term observational studies of NTG have indicated that progression of VFD

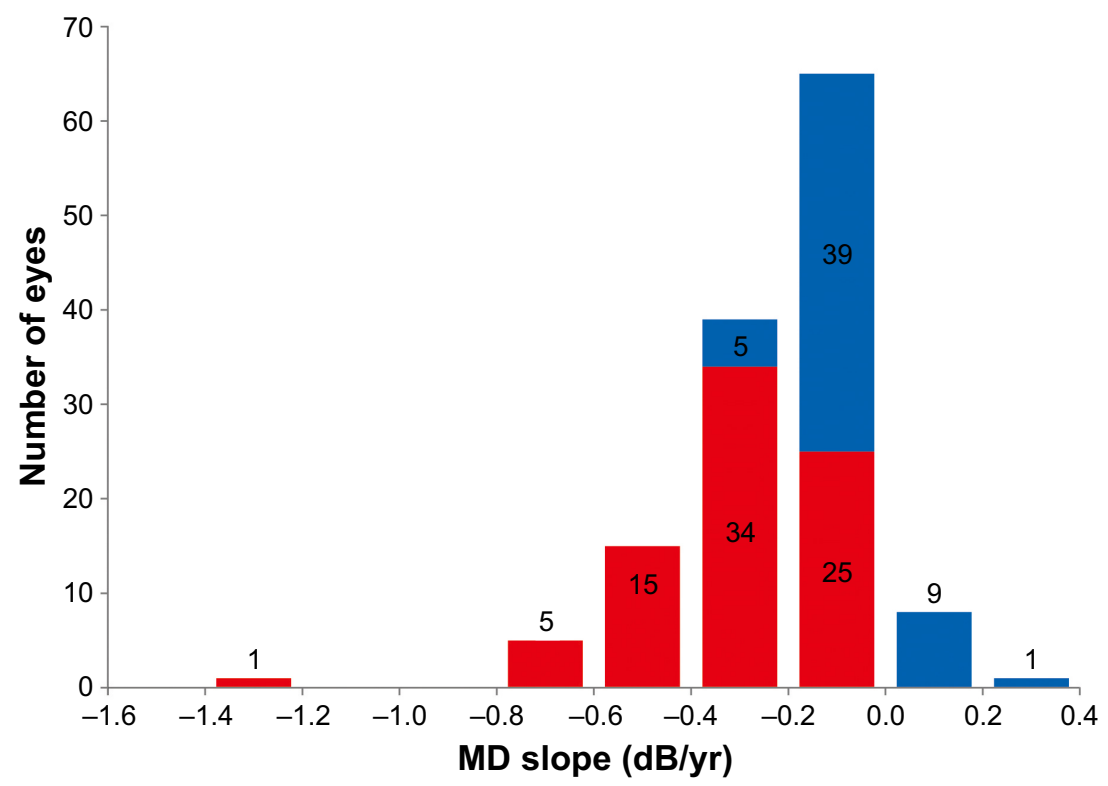

Figure 2 Distribution of number of eyes with MD slope value at the final visual field test.

Notes: At the final visual field test, significantly negative MD slope was observed in 80 (59.7\%: red column) of I34 eyes, but MD slope deterioration was not significant in 54 eyes (40.3\%: blue column). All 21 eyes with MD slope of lower than $-0.4 \mathrm{~dB} /$ year showed significant deterioration. Of $102 \mathrm{eyes}$ with MD slope was less than $-0.4 \mathrm{~dB} /$ year, however, 59 eyes (57.8\%) appeared to be significant MD slope deterioration.

Abbreviation: MD, mean deviation. 
Table 2 Results of trend analysis and univariate logistic regression analysis

\begin{tabular}{|c|c|c|c|}
\hline \multirow{3}{*}{$\begin{array}{l}\text { Consecutive } \\
\text { decrease } \\
\text { of MD }\end{array}$} & \multicolumn{2}{|l|}{ MD slope } & \multirow{3}{*}{$\frac{\text { Total }}{\mathrm{n}(\%)}$} \\
\hline & \multirow{2}{*}{$\begin{array}{l}\text { Deterioration } \\
\mathrm{n}(\%)\end{array}$} & \multirow{2}{*}{$\begin{array}{l}\text { Nondeterioration } \\
\mathbf{n}(\%)\end{array}$} & \\
\hline & & & \\
\hline$\leq 1$ & $2(50.0)$ & $2(50.0)$ & $4(3.0)$ \\
\hline 2 & $23(40.4)$ & $34(59.6)$ & $57(42.5)$ \\
\hline 3 & $26(66.7)$ & $13(33.3)$ & $39(29.1)$ \\
\hline$\geq 4$ & $29(85.3)$ & $5(14.7)$ & $34(25.4)$ \\
\hline \multirow[t]{3}{*}{ Total } & $80(59.7)$ & $54(40.3)$ & 134 \\
\hline & & & $P$-value: \\
\hline & & & $<0.0001$ \\
\hline Value & \multicolumn{2}{|c|}{ Odds ratio $(95 \% \mathrm{Cl})$} & $P$-value \\
\hline Consecutive & \multirow{2}{*}{\multicolumn{2}{|c|}{$2.19(1.44-3.34)$}} & $<0.0001$ \\
\hline decrease of MD & & & \\
\hline
\end{tabular}

Notes: Objective variable: presence or absence of MD slope deterioration. Explanatory variables: consecutive deterioration of VFD.

Abbreviations: $\mathrm{Cl}$, confidence interval; IOP, intraocular pressure; $\mathrm{MD}$, mean deviation; VFD, visual field defects.

occurs in approximately $50 \%$ of patients $(46.3 \%-53 \%) .{ }^{8,10,14}$ Progression of NTG can also be assessed by morphological changes. ${ }^{15}$ However, trend analysis and calculation of the MD slope are generally employed for assessment of VFD, since

Table 3 Comparison of variables between groups with and without MD slope deterioration

\begin{tabular}{|c|c|c|c|}
\hline \multirow[t]{3}{*}{ Factor } & \multicolumn{2}{|l|}{ MD slope } & \multirow[t]{3}{*}{$P$-value ${ }^{a}$} \\
\hline & Deterioration & Nondeterioration & \\
\hline & $(n=80)$ & $(n=54)$ & \\
\hline Sex n (\%) & & & 0.4755 \\
\hline Female & $51(63.8)$ & $31(57.4)$ & \\
\hline Male & $29(36.2)$ & $23(42.6)$ & \\
\hline Age (years) & $57.6 \pm 10.88$ & $57.9 \pm 11.95$ & 0.8943 \\
\hline $\begin{array}{l}\text { Follow-up duration } \\
\text { (years) }\end{array}$ & $8.0 \pm 0.81$ & $8.0 \pm 0.86$ & 0.9792 \\
\hline Refractive error (D) & $-3.2 \pm 3.31$ & $-3.2 \pm 3.92$ & 0.9410 \\
\hline ССТ $(\mu \mathrm{m})$ & $5|8.5 \pm 3| .3 \mid$ & $523.5 \pm 34.84$ & 0.3935 \\
\hline $\mathrm{DH}, \mathrm{n}(\%)$ & & & 0.5265 \\
\hline+ & $19(23.8)$ & $10(18.5)$ & \\
\hline- & $61(76.2)$ & $44(81.5)$ & \\
\hline Baseline IOP (mmHg) & $14.7 \pm 1.95$ & $15.3 \pm 1.99$ & 0.0896 \\
\hline Mean IOP $(\mathrm{mmHg})$ & $12.0 \pm 0.85$ & $12.0 \pm 0.92$ & 0.5307 \\
\hline IOP reduction rate (\%) & $17.2 \pm 8.64$ & $21.2 \pm 7.68$ & $0.0053^{*}$ \\
\hline $\begin{array}{l}\text { IOP reduction range } \\
(\mathrm{mmHg})\end{array}$ & $-2.67 \pm 1.59$ & $-3.37 \pm 1.54$ & $0.0130 *$ \\
\hline Maximum IOP $(\mathrm{mmHg})$ & $14.3 \pm 1.54$ & $\mid 4.4 \pm 1.76$ & 0.7797 \\
\hline Minimum IOP (mmHg) & $10.3 \pm 0.93$ & $10.4 \pm 1.05$ & 0.5660 \\
\hline IOP range $(\mathrm{mmHg})$ & $4.0 \pm 1.24$ & $4.0 \pm 1.34$ & 0.9340 \\
\hline IOP fluctuation $(\mathrm{mmHg})$ & $1.0 \pm 0.21$ & $1.0 \pm 0.24$ & 0.5765 \\
\hline Initial MD value $(\mathrm{dB})$ & $-7.1 \pm 5.6 I$ & $-8.0 \pm 5.43$ & 0.3950 \\
\hline Final MD value $(\mathrm{dB})$ & $-|1.8 \pm 6.6|$ & $-8.3 \pm 5.77$ & $0.0016^{*}$ \\
\hline MD slope (dB/yr) & $-0.32 \pm 0.20$ & $-0.06 \pm 0.12$ & $<0.000 *$ \\
\hline
\end{tabular}

Notes: aWelch's t-test except sex, DH (Fisher's exact test); *Values indicate those results that are statistically significant at the $P<0.05$ level.

Abbreviations: CCT, central corneal thickness; $\mathrm{DH}$, disc hemorrhage; IOP, intraocular pressure; MD, mean deviation; SD, standard deviation.
Table 4 Results of multivariate logistic regression analysis

\begin{tabular}{lll}
\hline Value & Odds ratio (95\% Cl) & $P$-value \\
\hline Consecutive decrease of MD & $2.43(\mathrm{I} .55-3.80)$ & $<0.000 \mathrm{I}$ \\
IOP reduction rate (\%) & $0.92(0.88-0.97)$ & 0.0020 \\
\hline
\end{tabular}

Notes: Objective variable: presence or absence of MD slope deterioration. Explanatory variables: consecutive deterioration of VFD, IOP reduction rate versus baseline.

Abbreviations: $\mathrm{Cl}$, confidence interval; IOP, intraocular pressure; $\mathrm{MD}$, mean deviation; VFD, visual field defects.

the definition of progression according to morphological data has not been fully established. According to a definition of VFD deterioration as MD slope exceeding $0.5 \mathrm{~dB} / \mathrm{yr}$, Komori et $\mathrm{a}^{10}$ reported a frequency of VFD progression of $53 \%$. In the present study, incidence of VFD progression was $59 \%$, which was slightly higher than previous reports. ${ }^{8-11} \mathrm{We}$ defined VFD progression as a significant decrease in MD slope determined by regression analysis; mean MD slope value was $-0.32 \mathrm{~dB} / \mathrm{yr}$. Even in normal individuals, $\mathrm{MD}$ values decline by $0.06-0.08 \mathrm{~dB} / \mathrm{yr}$ with increasing age. ${ }^{16}$ VFD progression criteria in the present study, therefore, were considered appropriate for NTG patients under long-term observation. Trend analysis and the odds ratio of univariate logistic regression analysis for consecutive decreases of MD versus deterioration of MD slope was 2.26, indicating that the deterioration of the MD slope was significantly related to consecutive decrease in MD values. Thus, consecutive decreases in MD values predict subsequent worsening of VFD in NTG patients, and suggest the necessity of additional treatment.

$\mathrm{DH},{ }^{8,10}$ myopia, ${ }^{9}$ and $\mathrm{CCT}^{17}$ are documented risk factors for VFD progression in NTG. However, some studies have shown these factors to be unrelated to VFD progression..$^{14,18-20}$ In our current study, no apparent difference was observed among these factors when compared between groups (eyes with deterioration of MD slope and those with nondeterioration). The multiple disparate pathologies of NTG may be responsible for varying results among different studies.

Factors related to IOP, in contrast, have shown consistent relationships to the progression of VFD in most previous reports. However, there are differences among IOP-related factors, ie, peak IOP, ${ }^{14}$ changes in IOP ${ }^{21}$ or the IOP reduction rate, ${ }^{8}$ and their degree of influence over VFD progression. In our study, only IOP reduction rate was significantly greater in patients without MD slope deterioration than in those with deterioration.

Long-term studies of NTG have reported baseline IOPs of $15-16 \mathrm{mmHg}$ and mean IOPs during the observation 
period of $13-14 \mathrm{mmHg} .{ }^{8-10,14}$ In the present study, baseline IOP was not significantly different between the MD slope deterioration group and the nondeterioration group (14.7 $\mathrm{mmHg}$ versus $15.4 \mathrm{mmHg}$ ), but the rates of IOP reduction during the observation period were significantly different between groups. However, the numerical difference in rates was not large $-17.2 \%$ IOP reduction rate in the MD slope deterioration group and $21.5 \%$ in the nondeterioration group - suggesting that other factors may need to be considered. Accordingly, we conducted multivariate logistic regression analysis to statistically examine the relationships among variables that were significant according to univariate analysis. Since this was a retrospective observational study, we calculated the odds ratio rather than the risk ratio. Our results demonstrated that progression of VFD was related to the number of consecutive decreases in MD value, while the IOP reduction rate was related to nondeterioration of VFD.

We also investigated predictions of future VFD deterioration in NTG patients with the naïve Bayes method. ${ }^{22}$ Assuming that consecutive decreases in MD values and reduction of IOP occur independently, 10,000 simulations were conducted, and a classification rule (deterioration/ nondeterioration) was prepared. From this, the concordance rate was $69.8 \%$ and its $95 \%$ CI was $69.6 \%-70.0 \%$. Effective methods for predicting the progression of glaucomatous VFD have not yet been established. For example, the concordance rate of ophthalmologists for predicting progression of VFD is nearly $60 \%{ }^{23}$ Therefore, the present simulation was supportive of consecutive decreases in MD values and IOP reduction rate as prognostic factors for VFD progression in NTG patients.

\section{Conclusion}

In the present study population, a $20 \%$ reduction of IOP from baseline may slow the progression of VFD, a result that emphasizes the importance of controlling IOP in patients with NTG.

\section{Acknowledgments}

This was an investigator-industry collaborative study, funded by Santen Pharmaceutical Co., Ltd. (Osaka, Japan). Santen Pharmaceutical Co. Ltd. participated in the design of the study, suggestions of the statistical method, interpretation of the data, and preparation and review of the manuscript, but had no role in the collection and analysis of data.

\section{Disclosure}

Santo K, Hizaki H, and Hashimoto M are employees of Santen Pharmaceutical Co., Ltd. The authors report no other conflicts of interest in this work.

\section{References}

1. Heijl A, Lindgren G, Olsson J. A package for the statistical analysis of visual fields. Doc Ophthalmol Proc Ser. 1987;49:153-168.

2. Chauhan BC, Garway-Heath DF, Goñi FJ, et al. Practical recommendations for measuring rates of visual field change in glaucoma. $\mathrm{Br} J$ Ophthalmol. 2008;92(4):569-573.

3. Crabb DP, Garway-Heath DF. Intervals between visual field tests when monitoring the glaucomatous patient: wait-and-see approach. Invest Ophthalmol Vis Sci. 2012;53(6):2770-2776.

4. Naito T, Yoshikawa K, Mizoue S, et al. Relationship between consecutive deterioration of mean deviation value and progression of visual field defect in open-angle glaucoma. Clin Ophthalmol. 2015;9:2217-2222.

5. Chandler PA. Long-term results in glaucoma therapy. Am J Ophthalmol. 1960;49(2):221-246.

6. Grant WM, Burke JF Jr. Why do some people go blind from glaucoma? Ophthalmology. 1982;89(9):991-998.

7. Brubaker RF. Delayed functional loss in glaucoma. LII Edward Jackson Memorial Lecture. Am J Ophthalmol. 1996;121(5):473-483.

8. Kim M, Kim DM, Park KH, Kim TW, Jeoung JW, Kim SH. Intraocular pressure reduction with topical medications and progression of normaltension glaucoma: a 12-year mean follow-up study. Acta Ophthalmol. 2013;91(4):e270-e275.

9. Sakata $\mathrm{R}$, Aihara $\mathrm{M}, \mathrm{Murata} \mathrm{H}$, et al. Contributing factors for progression of visual field loss in normal-tension glaucoma patients with medical treatment. J Glaucoma. 2013;22(3):250-254.

10. Komori S, Ishida K, Yamamoto T. Results of long-term monitoring of normal-tension glaucoma patients receiving medical therapy: results of an 18-year follow-up. Graefes Arch Clin Exp Ophthalmol. 2014;252(12): 1963-1970.

11. Lee J, Kong M, Kim J, Kee C. Comparison of visual field progression between relatively low and high intraocular pressure groups in normal tension glaucoma patients. J Glaucoma. 2014;23(8):553-560.

12. Kitazawa Y, Shirato S, Yamamoto T. Optic disc hemorrhage in lowtension glaucoma. Ophthalmology. 1986;93(6):853-857.

13. Viswanathan D, Goldberg I, Graham SL. Relationship of change in central corneal thickness to visual field progression in eyes with glaucoma. Graefes Arch Clin Exp Ophthalmol. 2013;251(6):1593-1599.

14. Erdem E, Williams A, Kuchar SD, Waisbourd M, Spaeth GL. Longterm ( $>8$ years) evaluation of progression in patients with low-pressure glaucoma. Eur J Ophthalmol. 2015;25(6):490-495.

15. Banegas SA, Antón A, Morilla A, et al. Evaluation of the retinal nerve fiber layer thickness, the mean deviation, and the visual field index in progressive glaucoma. J Glaucoma. 2016;25(3):e229-e235.

16. Suzumura H, Yoshikawa K, Mizoue S, Hyodo R, Kimura T. Assessment of retinal sensitivity using a time-saving strategy in normal individuals. Clin Ophthalmol. 2012;6:1873-1878.

17. Gordon MO, Beiser JA, Brandt JD, et al. The Ocular Hypertension Treatment Study: baseline factors that predict the onset of primary open-angle glaucoma. Arch Ophthalmol. 2002;120(6):714-720.

18. Mitchell P, Hourihan F, Sandbach J, Wang JJ. The relationship between glaucoma and myopia: the Blue Mountains Eye Study. Ophthalmology. 1999;106(10):2010-2015.

19. Medeiros FA, Sample PA, Zangwill LM, Bowd C, Aihara M, Weinreb RN. Corneal thickness as a risk factor for visual field loss in patients with preperimetric glaucomatous optic neuropathy. Am J Ophthalmol. 2003;136(5):805-813.

20. Sohn SW, Song JS, Kee C. Influence of the extent of myopia on the progression of normal-tension glaucoma. Am J Ophthalmol. 2010;149(5): 831-838. 
21. Fukuchi T, Yoshino T, Sawada H, et al. The relationship between the mean deviation slope and follow-up intraocular pressure in open-angle glaucoma patients. J Glaucoma. 2013;22(9):689-697.

22. Yager RR. An extension of the naive Bayesian classifier. Inf Sci. 2006;176(5):577-588
23. Lin AP, Katz LJ, Spaeth GL, et al. Agreement of visual field interpretation among glaucoma specialists and comprehensive ophthalmologists: comparison of time and methods. Br J Ophthalmol. 2011;95(6):828-831.

\section{Publish your work in this journal}

Clinical Ophthalmology is an international, peer-reviewed journal covering all subspecialties within ophthalmology. Key topics include: Optometry; Visual science; Pharmacology and drug therapy in eye diseases; Basic Sciences; Primary and Secondary eye care; Patien Safety and Quality of Care Improvements. This journal is indexed on

Submit your manuscript here: http://www.dovepress.com/clinical-ophthalmology-journal

\section{Dovepress}

PubMed Central and CAS, and is the official journal of The Society of Clinical Ophthalmology (SCO). The manuscript management system is completely online and includes a very quick and fair peer-review system, which is all easy to use. Visit http://www.dovepress.com/ testimonials.php to read real quotes from published authors. 